\title{
A MULTI-PURPOSE VISION-EQUIPPED-REMOTELY-OPERABLE RIG FOR HYDRO-UNITS MONITORING
}

\author{
Ion Rares Stanciu ${ }^{1, a}$, Gheorghita Ginga ${ }^{1, b}$, Sebastian Muntean ${ }^{2}$ \\ and Liviu E. Anton ${ }^{1, c}$ \\ ${ }^{1}$ Politehnica University, Timisoara, Victoriei Square 2, 300006, Timisoara, Romania \\ ${ }^{2}$ Centre of Advanced Research in Engineering Sciences, Romania Academy-Timisoara Branch, \\ Bv. Mihai Viteazu 24, 300223, Timisoara, Romania \\ arares.stanciu@mh.mec.upt.ro, bpuiuginga@mh.mec.upt.ro, seby@acad-tim.tm.edu.ro, \\ cliviu.anton@tiriacauto.ro
}

\begin{abstract}
Keywords: Storage Pumping Stations, Centrifugal Pumps, Induction Motors, Remote Control, IPCamera.
\end{abstract}

\begin{abstract}
Hydroelectricity is a viable resource for production today. Being renewable, delivering system services and producing the cheapest electricity possible, these are facts that recommend hydroelectricity today. Oftentimes power plants work together with storage pumping stations. Simulation and multiple-speed experimental testing work together to ensure their efficiency increase when redesigning and/or refurbishing. Very important also is the operating cost reduction. Eliminating cavitation regimes lead to a longer life for the machine. The net result is a reduced maintenance activity therefore cost cuts. Operating these stations remotely, offer the possibility to reduce the operating and maintenance costs. Data acquisition feature helps managers to better schedule the maintenance activity with the net effect of reducing the operation cost. This paper presents a testing platform retrofitted with remote control capability and camera vision to demonstrate the remote control and monitoring. Built in the lab, the experimental facility is a valuable hardware platform allowing the researcher to target different research directions.
\end{abstract}

\section{Introduction}

The electricity is today mass-produced using: nuclear, thermal and hydro. The first two work in a similar manner: steam forces the turbine-generator bundle into rotation. On the other hand, the hydro uses water to produce electricity. According to [1] 20\% of world's electricity is delivered by hydro (in Romania, this percentage is around 30\%). Hydroelectricity advantages are: the price (it is the cheapest electricity), the absence of pollution, and its renewable character.

The need for electricity is far from constant over the time. During daytime the need for electricity is high while during the night time it decreases considerably. The demand-pattern varies during the day of the week and during the season. Since the electricity cannot be stored, there is an obvious need to adjust the production in order to ensure the production of the needed amount of power. Taking into account that nuclear-based and thermal-based plants allow small adjustments only, it is up to the hydro to ensure them (they are usually known as system services). Due to the variation of the demand, the energy price varies as well. Ensuring system services means that hydro has to be able to produce the energy (when there is demand - during day time) and to absorb the energy when there is no demand (during night time). Indeed, it is possible to use electricity to pump the water back into the lakes using an extra pump. In this regard, the engineers have designed the so-called pump-turbine units (a single unit able to operate as a turbine or a pump). Storage pumps may be used just as well to pump water back into the lakes. Such an ensemble offers a more intelligent way of operation which has a significant economic advantage. The rapid increase of energy price during the last decades has created the need for new control ways able ensure cost reduction. An increasingly important source of revenue is the so-called energy forecasting [2]. 
Another form of electricity is gaining importance at fast pace: the wind energy. Being free is its main advantage. However its integration into the power system poses a significant challenge due to its intermittent character. In an attempt to protect the power grid stability, a significant number of islands have imposed restrictions on direct penetrations of wind power according to Bueno and Carta [3]. Luickx [4] et al, indicate storage plants for wind integration. From all examined storage types (compressed air, fuel cells, flywheels batteries, heat pumps, and pumped hydro energy storage) they recommend the Heat Pumps (HPs) and the Pumped Hydro Energy Storage (PHES). Wind power integration using a cell-type structure consisting of wind turbine and PHES has been proved in [5].

Increasing the power plants off-design efficiency, modeling and testing is perhaps the most targeted research domain. Savar el al [6] is concerned with increasing the pumping efficiency by trimming its impeller. Their experimental tests have targeted a set of seven impeller diameters. Reducing operation cost can be achieved also by avoiding the so-called cavitation regime which is responsible in time for impeller destruction. This destructive regime is responsible for often maintenance, repairs, and dead times.

Another direction targets the operation cost reductions. Controlling the plant remotely allows cost reduction. This idea is not new. The implementation however, differs from application to application. Bekiroglu and Daldal [7] control remotely an ultrasonic motor. Using a tone decoder and the GSM mobile phone network they are able to control the speed, position and direction of the motor. Abdul Aziz et al [8] also employed the GPS infrastructure to remotely monitor an agricultural greenhouse using the Short Message Service (SMS). Their remote monitoring temperature system is able to alert the personnel regarding temperature changes. Bashkar and Manohar [9] use GSM to build a remote motor controller. The system they've built can control up to 8 devices by sending SMS from a mobile phone. Tan et al [10] presents an internet-based which allows process-monitoring from a distributed control system. Ko et al [11] describes a web-based virtual lab for teaching an undergraduate class. Swamy et al [12] have developed web-based automation. Al-Ali et al [13] introduced a Java-based, low-cost remote control for home automation.

Unlike the middle-size power plants which usually require personnel to operate, storage pumping stations may function without operators. Their simple task (turn on and off) allow remote control. A remote operation which includes turning on and off and data recording will decrease operating costs. Processing the recorded data allows a more efficient maintenance schedule and the use of a single team to maintain all the plants.

This paper presents a vision-equipped-remotely-operable experimental facility retrofitted with remote control and video for hydro-units testing. Section 2 discusses the infrastructure. Section 3 describes the remote control system. Using a client-server backbone an application is being created to completely and remotely control the experimental facility. A video system was also implemented. Its serves for two different purposes: deliver images from the station and for monitoring. So far only one camera is used. However multiple cameras can be installed. The images can be recorded on the computer and reviewed once needed. The video system is described in Section 4. Conclusions are drawn in the last section.

\section{The experimental facility}

Testing is used by researchers for various meanings. Validating their numerical results is an important purpose. The numerical analyses results are compared against the experimental ones to assess them. Faults can also be diagnosed through testing.

This experimental facility developed in the Pumps Laboratory at "Politehnica" University of Timisoara for testing purpose can be seen in Fig. 1. It is composed of a closed hydraulic circuit, two reservoirs of each, vanes, a PCN 80-200 pump, sensors (for pressure, temperature, discharge and electrical power), and data acquisition system to acquire sensors data. The inlet and outlet pipes diameters are $0.1 \mathrm{~m}$ and $0.08 \mathrm{~m}$, respectively. The pressure sensors height with respect to the pump are $\mathrm{Zasp}=0.01 \mathrm{~m}$ and $\mathrm{Zref}=0.75 \mathrm{~m}$. To be able to perform measurements and record operating data, an acquisition system was built. The features of interest are the inlet and outlet pressures, the speed, 
the discharge, the electrical power and the fluid temperature. Called "SES-A1" and built as a distinct module, it has 32 channels (with voltage/current differential inputs), 12 bits resolution, $100 \mathrm{~kb} / \mathrm{sec}$ acquisition frequency, 512k sample memory and allows computer connection. The Electronic Interface module provides the compatibility needed for computer interfacing using the RS232 standard. This board allows the adaptation to the input signal levels of the sensors.

A $37 \mathrm{KW}$ asynchronous motor was chosen to actuate the rig's pump. Its parameters can be seen in Table 1. This actuator is the industry's workhorse today due to its constructive simplicity, the absence of brushes and the low-cost maintenance, feature that make it the preferred actuator. Its single drawback is related to the speed adjustment. Fortunately, an inverter sidesteps this drawback. The actuation data is reported by the inverter which powers the motor. The hydraulic and actuation data is sent to the remote controller which may use it to estimate the hardware condition in order to schedule maintenance.

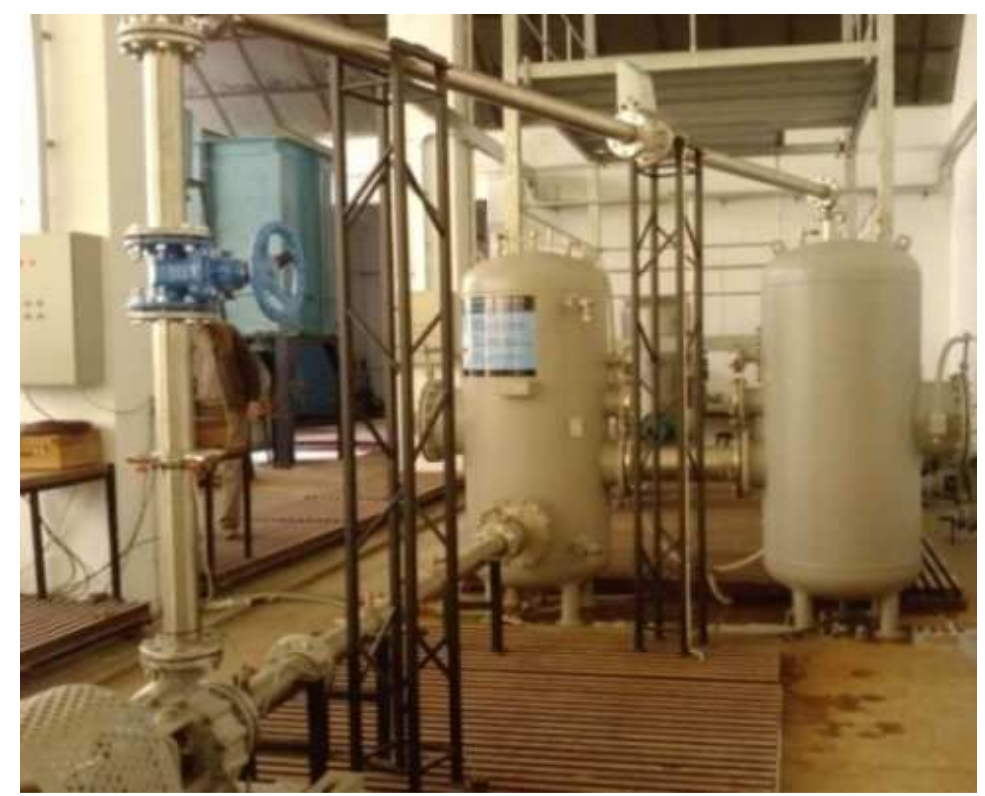

Fig. 1 - The experimental facility in the lab
Table 1: The motor parameters

\begin{tabular}{|c|c|}
\hline Parameter (nominal) & Values \\
\hline Power (at shaft) [W] & 37000 \\
\hline Voltage [V] & 400 \\
\hline Current [A] & 66.58 \\
\hline Efficiency [\%] & 89.64 \\
\hline Power Factor & 0.895 \\
\hline Speed [rpm] & 2958 \\
\hline Torque [Nm] & 119.56 \\
\hline Slip [\%] & 1.4 \\
\hline
\end{tabular}

To be able to efficiently adjust the flow rate, a Direct Torque Control (DTC) inverter was installed. The DTC technology allows large speed adjustments and thus, many different values of flow rate. This approach was preferred after examining several possibilities (gearbox, motor-generator group, etc.) and taking into consideration the already available actuators.

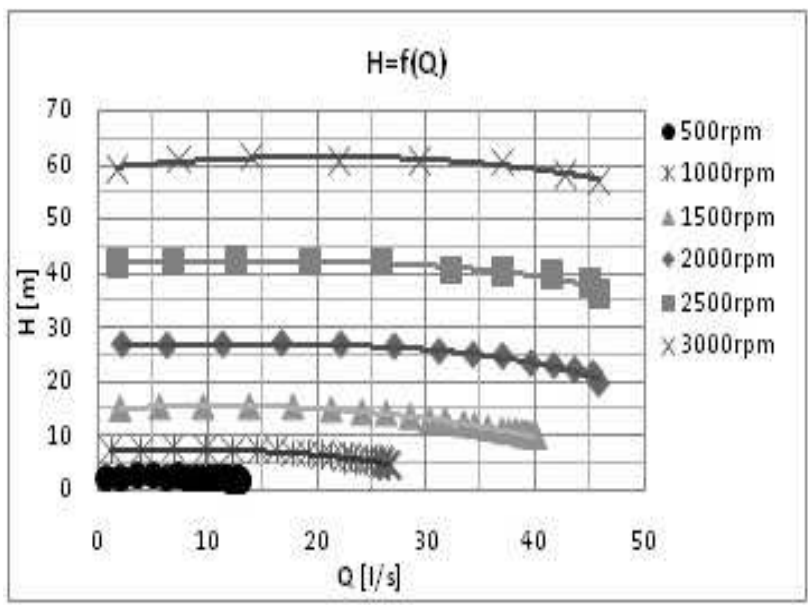

Fig. 2 - The $H=f(Q)$ for several speed values for our infrastructure's pump
Adjusting remotely the actuation speed comes handy when storage pumping stations will be equipped with high-power inverters. Despite using the DTC control, the system revealed torque pulsations when operating at low speed values (500rpm). These ripples generate the so-called "noise" which can be found in the speed, the mechanical power, the electrical power, and the efficiency curves. A Kalman filter was successfully implemented in the previous work to eliminate these ripples [14].

A software platform ensures the complete control of the experimental facility. Developed inside the lab, it acquires the variables (the motor's speed, the temperature, the inlet and outlet pressures, and the discharge) and stores them in an Excel file. Having real-time plotting capability (it contains a graphical area which plots the $H=f(Q))$ the platform performing all the necessary calculations. A $H=f(Q)$ family of curves for multiple speed values can be seen in Fig 2. Its Graphical User Interface (GUI) displays the instantaneous values received from the SES-A1. The platform was developed in C\#. 
To be able to successfully control the inverter a Modbus communication module was integrated into the software platform. Modbus messages are being built and sent to the inverter to set the speed, start or stop the motor or to periodically read the parameters of interest (the speed, the current, the electrical and mechanical power). Modbus-related information is also displayed by the software platform's GUI (the slave address, the accessed register, the function code and the data retrieved from inverter). Every message accesses a specific register in the inverter. The Modbus functions 06 - writing to a single register (for start/stop and setting the speed reference) and 03 reading a single register (to read values for speed, current, mechanical power delivered at motor's shaft and the electrical power received from the network) are used to control the rig.

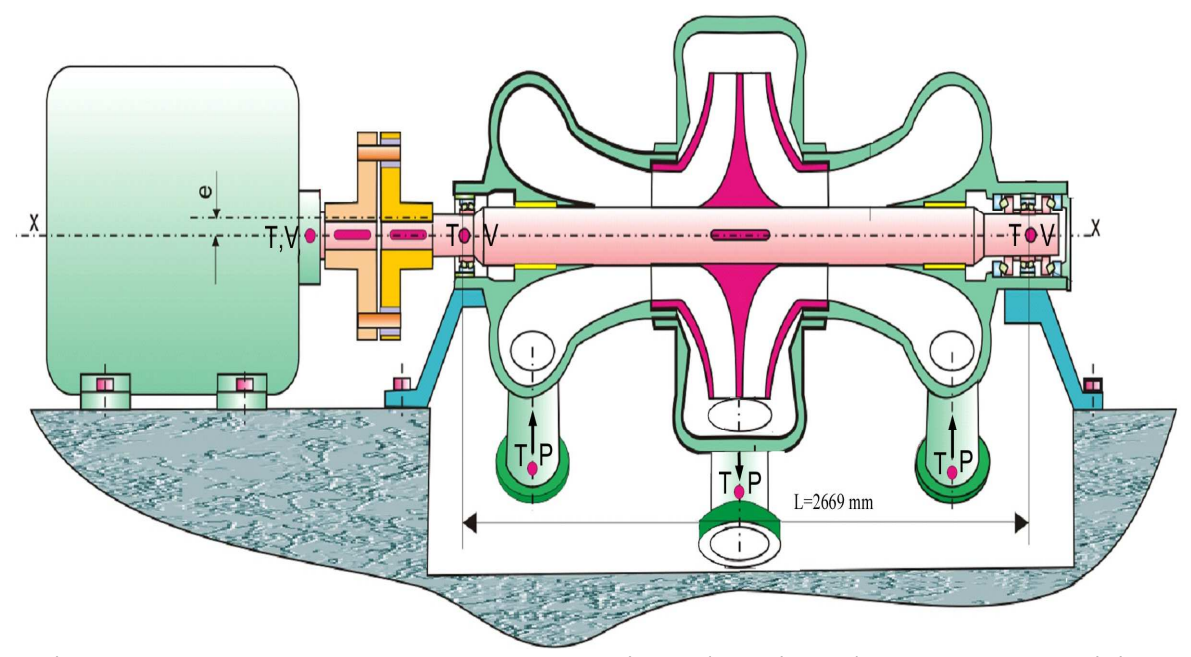

Fig. 3 - A storage pumps cross-section showing the sensors position (TP - for pressure, $\mathrm{T}$ - for temperature, $\mathrm{V}$ - for vibrations)

Several sensors have to be used to deliver monitoring data. Their placement on a storage pump can be seen in Fig. 3 . For a successful remotecontrol there are several important categories of parameters: hydraulic (the inlet and the outlet pressures, the flow-rate), actuation (the electrical and the mechanical power, the speed, and the current). Another parameter of interest is the temperature (these sensors deliver information regarding the bushes temperature). Vibration information is also used for monitoring.

Our experimental facility has been equipped with sensors for pressure, flow-rate, and temperature. The actuation parameters are being measured through the inverter.

\section{The remote controller}

The remote control is an increasing important direction in automation. Starting during the space exploration era, it becomes more and more important today. The application sphere has also grown: from space shuttles to autonomous robots (drones, home automation, and plant control. Many of the remote-control implementations prefer the use of GSM infrastructure. An important advantage of such an implementation is the wide GSM availability. However, because of their remote locations it is possible to be uncovered by cellular networks.

Internet support was preferred when developing this automation structure. The fact that no interfacing modules are required is an important advantage of this solution. Indeed, interfacing modules (not available at the moment) are needed to implement the remote control using the cellular network. Another advantage of using the Internet is represented by the implementation of the vision system (which is this work uses IP-cameras).

The remote controller described here is built using a client-server backbone. In this approach, two modules are required to function: a client and a server. In such an application, the server listens while de client attempts a connection. The server was implemented in the software platform that controls the rig. The structure of a remotely controlled application can be seen in Fig. 4. As it can be seen, the physical support for the remote controller can be a laptop/PC, or a Pocket PC (PPC). The remote controller sends commands to the PC in control of the plant. After indentifying them (parsing them) the local computer issues plant commands accordingly. Several commands were designed in order to achieve client-server communication. Together with their action, they are presented in Table 2 . 
The remote control mechanism is composed of a set of commands, two parsers (on each side), and the client-server mechanism. A detailed structure of this mechanism can be seen in Fig. 5. Several commands have been implemented to ensure remote control (the ones shown in Table 2 were designed to prove the concept of pump station remote operation). To distinguish between commands, a parser is being integrated in the software platform's code along with the webserver.

The "startmotor" command forces the software platform to instruct the inverter to start the motor (basically starting the pump). The "stopmotor" command works similarly and stops the rig's pump. The "increasespeed" and the "decreasespeed" instruct the software platform to add or subtract a constant value to the reference speed and submit the result to the inverter to modify this value. The "startautoacq" and "stopautoacq" commands instruct the software platform to start and stop data acquisition. The "getdata" command instructs the software platform to submit the acquisition data to the controller.

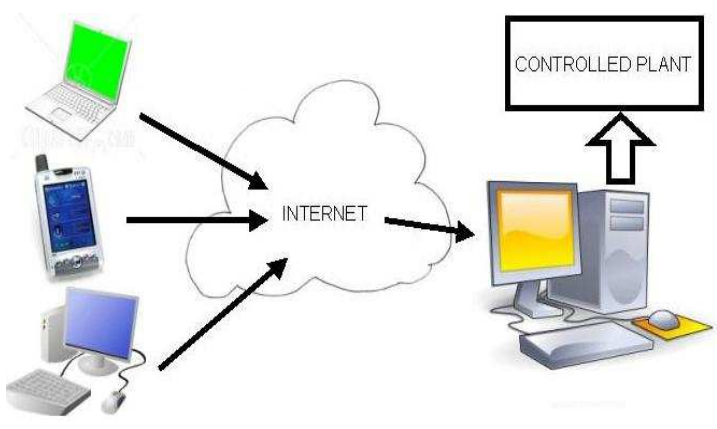

Fig. 4 - The remote control

Table 2: The remote control commands

\begin{tabular}{|c|c|}
\hline Commands & Action \\
\hline "startmotor" & Turns on the pump motor \\
\hline "stopmotor" & Turns off the pump motor \\
\hline "increasespeed" & $\begin{array}{c}\text { Increase the motor speed by certain } \\
\text { amount of rpm }\end{array}$ \\
\hline "decreasespeed" & $\begin{array}{c}\text { Decrease the motor speed by } \\
\text { certain amount of rpm }\end{array}$ \\
\hline "startautoacq" & $\begin{array}{c}\text { Starts the data acquisition } \\
\text { "stopautoacq" }\end{array}$ \\
\hline "getdata" & $\begin{array}{c}\text { Retrieves the acquisition data } \\
\text { which are sent to the remote } \\
\text { controller }\end{array}$ \\
\hline "setacqinterval" & Modifies the acquisition interval \\
\hline
\end{tabular}

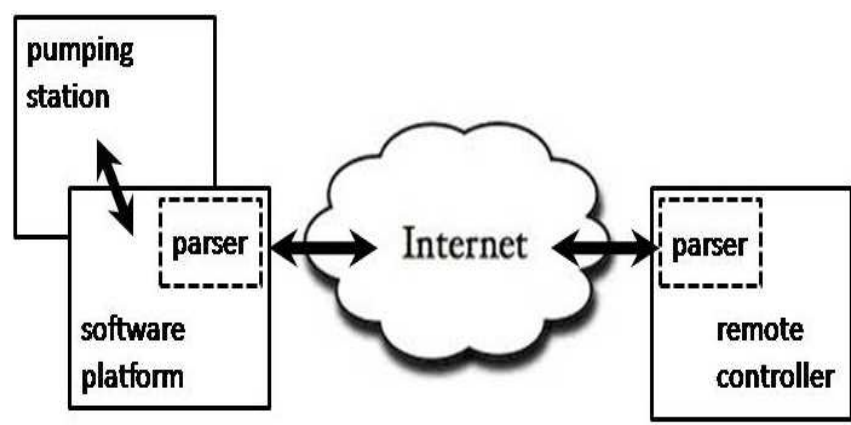

Fig. 5: The controller's structure

When a "getdata" command is received the software platform constructs a string with the values for the motor speed (reference and actual values), the electrical and mechanical power, the input and output pressure, the flow-rate, the head, the efficiency, and the acquisition interval. To make the parsing easier, each parameter is preceded by its name and followed by its measuring unit. The sobuilt string gets sent to the remote controller. After parsing, the controller displays the data in the screen and logs it into a file for later analysis. The "setacqinterval" command is the only one taking a parameter: the sampling time (in seconds). Upon request, new commands can be added to the modules involved. The controller saves the received operation data into a file, thus allowing for later processing.

\section{The video system}

For research purpose and to better emulate the Jidoaia Pumping Station fluid flow at pump entrance a scaled down suction elbow was installed on the experimental platform. The elbow was made of transparent plexiglass, thus allowing for image capturing and future LDV measurements [15]. Using a camera, one can grab fluid flow images and compare them against simulation results.

Developing a vision system has two reasons. Firstly, during the experiments, taken images can qualitatively prove (or reject) numerical simulations. For this purpose, a scaled down plexiglassmade elbow was mounted on the experimental platform to better emulate the flow in the Jidoaia 
Pumping Station. Flow images can now be taken using a video system. Secondly, for a remote operation purpose, an image inside the storage pumping station offers a pretty good idea about the station's state. For example, mechanical failures (such as broken shafts, accidental floods, etc.) can be successfully detected using camera images.

Useful in any of the above-mentioned situations, the vision system was designed and implemented. This feature allows remote operators to identify possible problems in the station using vision. Using more cameras is possible and entirely up to the customer. Indeed a multi-camera system delivers more pumping station images (data). IP cameras were preferred due to their advantages (good resolution, reasonable price, remote controllability, etc). They can be accessed using HTTP commands. To be able to communicate with a camera (including receiving image) the user has to know several camera parameters: the camera IP address and port, the username and the password, etc. These parameters can be set using HTTP commands. The camera parameters to be set are shown in Table 3. Two IP-cameras cameras (fixed and PTZ) can be seen in Fig. 6.

\subsection{Detecting the IP cameras on the local network}

An important feature of the video system is the ability to perform a so-called "automatic setup", thus eliminating the need for IT personnel. To access an IP-camera one must know its parameters (IP-address, port, username/camera, etc.). For automatic setup purpose, the cameras are to be detected on the local network and configured in an automatic fashion [15]. When first connected to the router and powered, the cameras obtain IP-addresses assigned by the router. In order to be able to properly setup the camera, the user (or the program) has to know the IP-addresses of the cameras. An elegant way to detect cameras is to use the UPnP technology.

Table 3: Camera parameters

1) The "root" account setup,

2) New camera account setup (username and password),

3) Enable/disable anonymous view,

4) Enable/disable anonymous PTZ control,

5) Camera IP-address (when first powered, the camera receives an IP-address assigned by the router),

6) DHCP or fixed IP address,

7) Camera port (this is used when trying to access the camera from outside the local network),

8) Time/zone (the time has to be displayed on camera image),

9) Camera resolution, compression, and name
The UPnP standard defines the architecture for peer-to-peer network connectivity of PCs, networking devices and appliances. UPnP leverages the TCP/IP, UDP, HTTP and XML technologies, the objective being to enable networking, control and data transfer between the networked devices. The UPnP technology was employed in this paper to detect the IP-cameras on the local network. To detect cameras, the program uses the so-called UPnPDeviceFinderClass class from the UPNPLib available in Visual Studio 2005. Their specific functions are employed to detect the camera(s) and report their MAC and IP addresses.

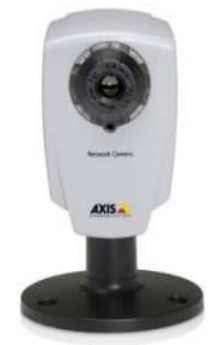

Fig. 6a) A fixed IP-camera

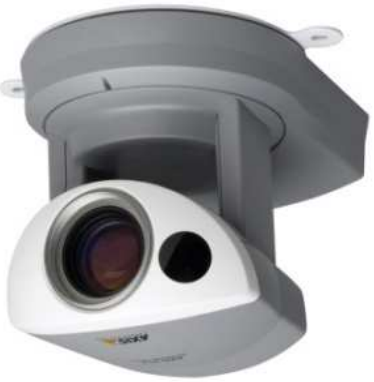

Fig. 6b) A PTZ IPcamera

\subsection{Setting up the IP cameras in an automatic fashion}

Once the cameras have been found on the local network, they can be set in a convenient way using HTTP commands. Each command changes one parameter after another. To change one parameter, the code has to know the camera's IP address and its port. This information is provided by the code responsible for camera detection. Once the IP and the ports are known, to access the camera the code builds the Uniform Resource Identifier (URI) 
using the UriBuilder class. After defining an UriBuilder variable, the command string is formed using the variables Path and Query. A request is created and sent to the camera using the HttpWebResponse class. A function responsible for sending data to the camera was implemented. Its schematic block diagram can be seen in Fig. 7. Every camera parameter (given in Table 3 just to give the reader an idea about the multi-camera system setup complexity) can be set using this structure. Because the strings that form the string differ from one command to another a set of functions was written (one for every HTTP command). Its schematic block diagram can be seen in Fig. 8. Each time a command is sent to the camera, the code examines the response. If the parameter has been set successfully, another command is being sent. An error is signalized if a command does not succed.

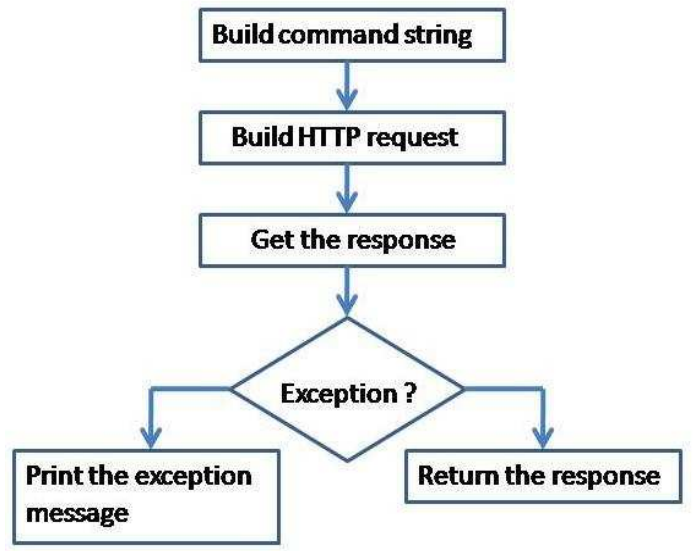

Fig. 7: The block diagram of the function responsible for sending HTTP commands

\section{Experiments and results}

With the experimental facility so equipped, several experiments were performed. The first one targeted the remote control. Commands were sent from the remote controller to the software platform to prove the remote controllability.

The second experiment was performed to visualize the hydrodynamic flow in the elbow and to identify instabilities types generated by the suction elbow. The experiment was videotaped using the camera (Fig. 9). A stroboscope was employed to light the elbow while videotaping. Two counter-rotating vortices were observed developing behind the shaft (Ginga et al [16] and Van den Braembusche et al [17]). The vortices can also be seen in Fig. 9.

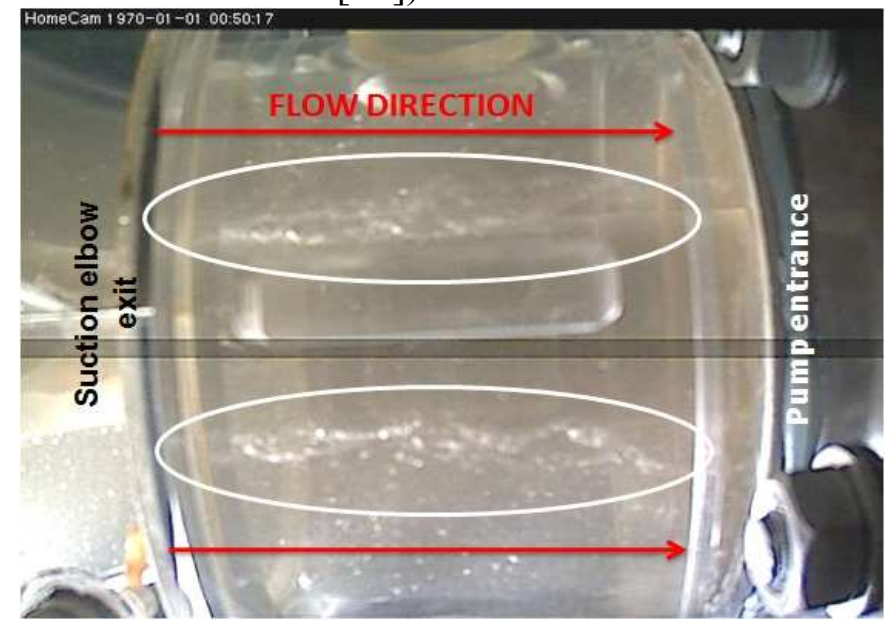

Fig. 9: A suction elbow snapshot reveals the two counter-rotating vortices

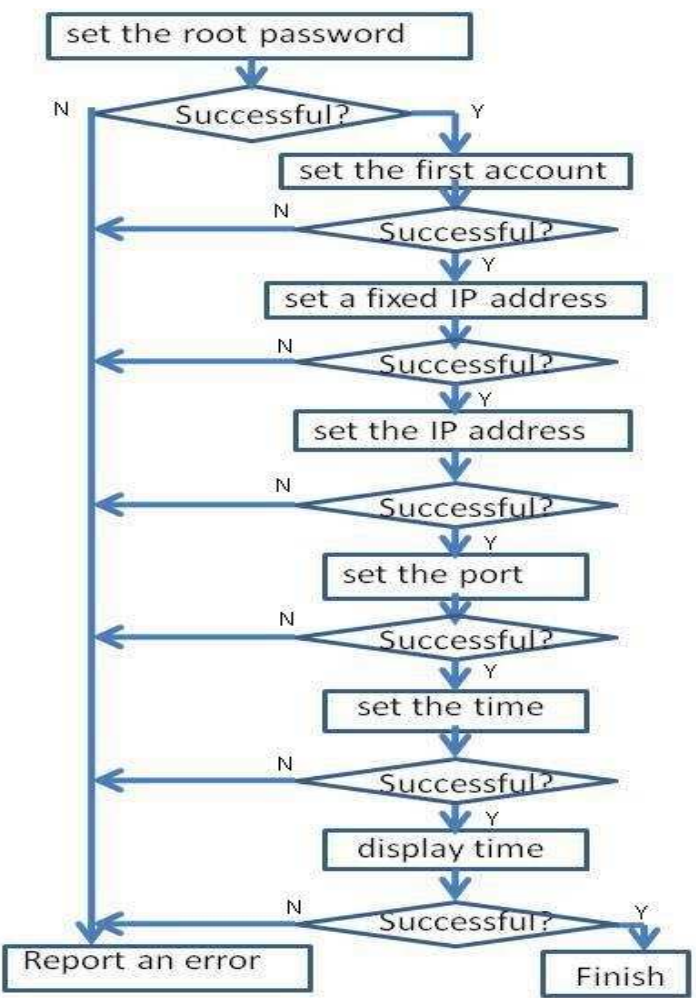

Fig. 8: The block diagram of the function responsible for camera setup
An analysis of one of the parameters of interest mentioned above was the objective of the third experiment and it is presented in this paragraph. Conventionally, the monitored parameter for hydrodynamic field is the pressure. Two pressure sensors (placed at 90 degrees) were currently employed for measurement. The reasons behind using two sensors are the availability of two sets of acquired data and the possibility to discriminate the unsteady pressure components. A schematic view of their position on the suction elbow can be seen in Fig. 10. The sensors mounted on the suction elbow can be seen in Figs. 11 and 12. 


\section{Innovation for Sustainable Development}

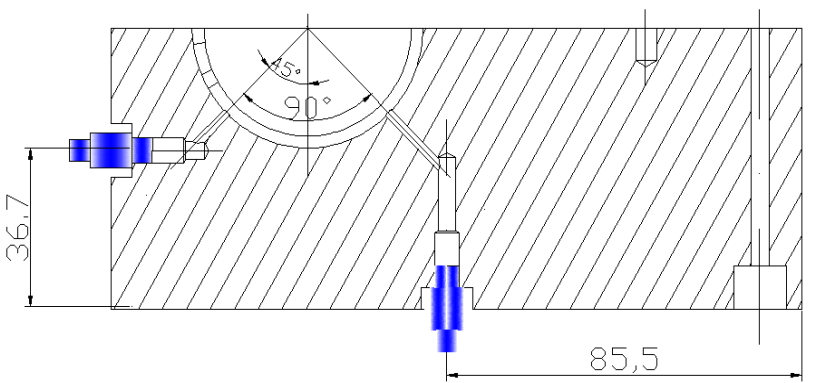

Fig. 10: The schematic showing the placement of the two sensors on the plexiglass elbow (view from the pump's entrance)
The unsteady pressure on the outlet elbow section corresponding to the pump's inlet leads to two components: the mean value and the pulsation. The mean value of the static pressure at the inlet of the pump is usually employed to assess the reference level. This value is used to determine the cavitational performance [18]. The pulsation component is used to determine the RMS value. This is directly related to the

dynamical behavior induced by the flow. An in-depth analysis of the dynamic behavior is performed using the discrimination between plunging and rotational components relays on the phase shift of the two pressure signals. The plunging wave is particularly dangerous because it propagates in the hydraulic circuit.
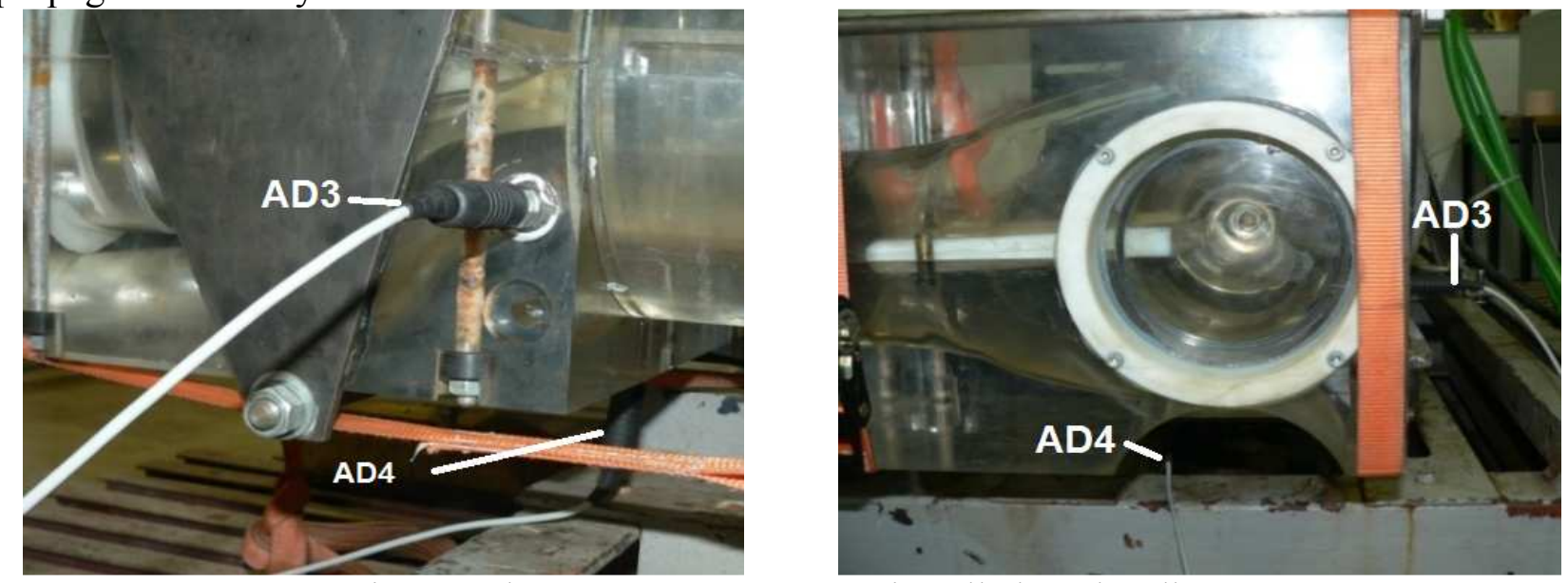

Fig. 11: The two pressure sensors installed on the elbow

The recorded experimental pressure pulsations corresponding to a speed of $3000 \mathrm{rpm}$ can be seen in Fig. 12. To be able to spot the important frequencies, its spectrum was plotted using the Fast Fourier Transform (FFT). The signal's spectrum can be seen in Fig. 13. Several peaks can be seen here. A peak representing the two vertices can be observed in the $0-50 \mathrm{~Hz}$ interval. Its frequency is $20 \mathrm{~Hz}$. The origin of the $50 \mathrm{~Hz}, 150 \mathrm{~Hz}$, and $250 \mathrm{~Hz}$ frequencies in Fig. 13 is the power network. It is also important to mention that the pump's impeller has 5 blades.

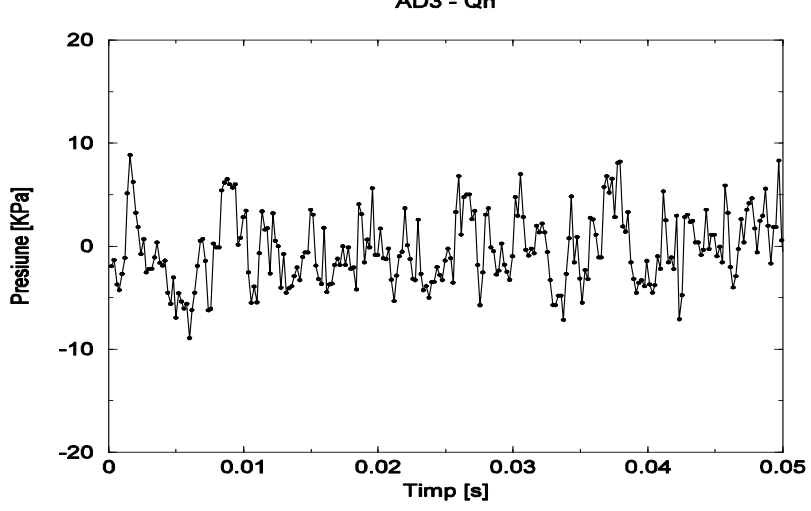

Fig. 12: The pressure fluctuation in the suction elbow at nominal flow-rate

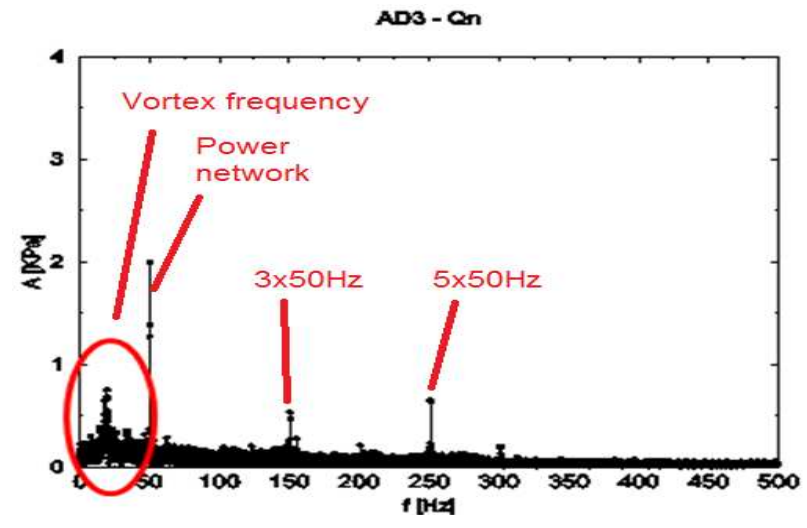

Fig. 13: The pressure signal spectrum at nominal flow-rate

Particularly to analyse the influence of the two vortices induced in the flow by the section elbow was performed in the frequency range of $0 \div 50 \mathrm{~Hz}$. A $15^{\text {th }}$ order Butterworth Low-Pass filter was implemented to eliminate the higher frequencies. The cut-off frequency was chosen to be $40 \mathrm{~Hz}$ in order to eliminate even the first power network frequency. The filtered signal can be seen in Fig. 14 (the black curve). The filtered signal was reconstructed using the Parceval's theorem and it can be 
seen in Fig. 14 (the red curve). The two spectra (for the filtered - black and reconstructed - red signals) can be seen in Fig. 15. Details related to the influence of these unsteady phenomena with hydraulic operating conditions are available in [19].

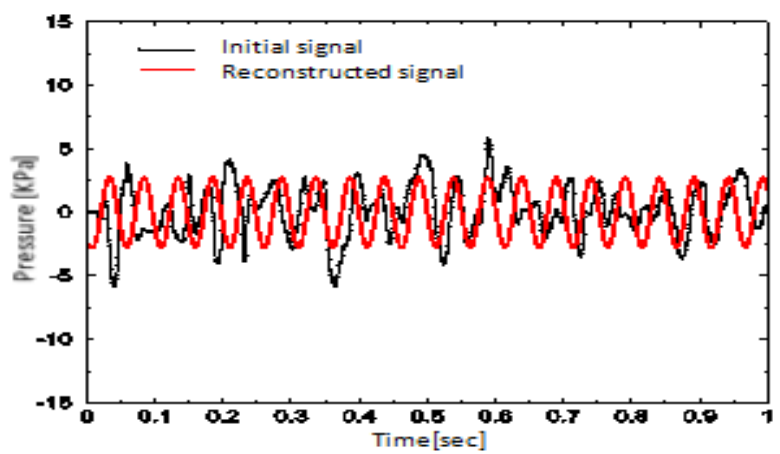

Fig. 14: The filtered and the reconstructed unsteady pressure signal at nominal flow-rate

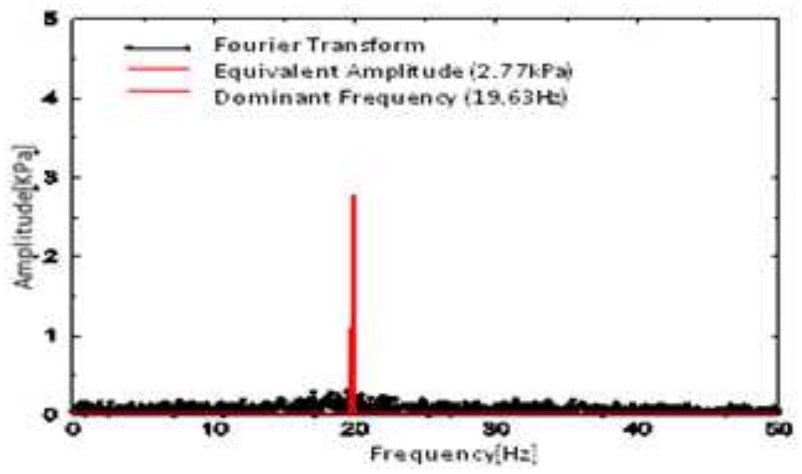

Fig 15: The filtered signal's spectrum at nominal flow-rate

\section{Conclusion and future work}

This paper presents a multi-purpose testing platform built in the Pumps lab at "Politehnica" University of Timisoara. Equipped with a variable speed actuator, data acquisition equipment, and sensors, the experimental facility is controlled by a software platform.

The objective of the latest work was a client-server application allowing the remote control of our experimental platform. A server module was embedded in this platform and a client was built to demonstrate the remote controllability for operating cost reduction purpose. The performed experiments have demonstrated the successful remote control. However, no protection mechanism was implemented (or tested) to prevent or signalize the loss of communication.

A video system was also developed. Taken images can prove (or not) the numerical simulation results. Live images can be recorded when operating remotely. An IP-camera was preferred to ensure an easy access. The experiments revealed the existence of two counter-rotating vortices in the suction elbow. The unsteady pressure was acquired. In an effort to determine the vortices frequency, the data was filtered using a low-pass filter. The filtered data was reconstructed using the Parceval's theorem.

\section{Acknowledgement}

This paper was supported by the project "Development and support for multidisciplinary postdoctoral programs in major technical areas of national strategy for Research - Development Innovation" 4D-POSTDOC, contract nr. POSDRU/89/1.5/S/52603, project co-funded by the European Social Fund through Sectorial Operational Program Human Resources Development 2007-2013.

Mr. Ginga's work was partially supported by the strategic grant POSDRU/88/1.5/S/50783 (2009) of the Ministry of Labor, Family and Social Protection, Romania, co-financed by the European Social Fund - Investing in people.

Dr. Sebastian Muntean was supported by the Romanian Academy program.

\section{References}

[1] Sternberg, R., "Hydropower: Dimensions of social and environmental coexistence", Renewable and Sustainable Energy Reviews, 12(6), 2008. pp.1588-1621

[2] Stanciu R., Sorandaru C., "Low-cost, short-term electric load prediction using the $\alpha-\beta-\gamma$ filter", Proc. of the 15th IEEE International Conference on Intelligent Engineering Systems, Poprad, Slovakia, 23-25 June, 2011. pp. 335-340, DOI: 10.1109/INES.2011.5954769.

[3] Bueno, C., Carta, J., A., "Wind powered pumped hydro storage systems, a mean of increasing the penetration of renewable energy in the Canary Islands", Renewable and Sustainable Energy Reviews, Vol. 10, 2006. pp. 312-340 
[4] Luickx P., J., Delarue, E., D., D'Haeseleer W., D., "The Examination of Different Energy Storage Methods for Wind Power Integration” TME Working Paper. Katholieke Universiteit Leuven, TME, 2008.

[5] Stanciu, I., R., Turcin, I., Muntean, S., Anton, L., E., "Cellular Wind-Power Integration using Remotely Controlled Pump Hydro Energy Storage", Proceedings of the Romanian Academy Journal, Series A. (accepted)

[6] Savar, M., Hrvoje, K., Sutlovic, I., "Improving centrifugal pump efficiency by impeller trimming", The International Journal on the Science and Technology of Desalting and Water Purification, 249, 2009. pp. 654-659

[7] Bekiroglu, E., Daldal, N., "Remote control of an ultrasonic motor using a GSM mobile phone", Sensors and Actuators A, 120, 2005. pp. 536-542

[8] Aziz, I., A., Hasan, M., H., Ismail, M., J., Mehat, M., Haron, N., S., "Remote Monitoring in Agricultural Greenhouse Using Wireless Sensor and Short Message System (SMS)", International Journal of Engineering \& Technology IJET Vol. 9, No. 9, 2009. pp. 1-12,

[9] Bhaskar, V., Manohar, T., G., "GSM Based Motor Monitoring and Speed Control", International Journal of Mechanical and Industrial Engineering (IJMIE), ISSN No. 2231 6477, Vol. 1, Issue 2, 2011.

[10] Tan, K., T. Lee, T., and Yee Soh, C., "Internet-Based Monitoring of Distributed Control Systems-An Undergraduate Experiment", IEEE Transactions on Education, Vol. 45, No. 2, May 2002. DOI: 10.1109/TE2002.1013876,

[11] Ko, C., C., Ben M. Chen, Hu, S., Ramakrishnan, V., Cheng, C., D.,, Zhuang, Y., and Chen, J., "A Web-Based Virtual Laboratory on a Frequency Modulation Experiment," IEEE Transactions on Systems, Man, and Cybernetics-Part C: Application and Reviews, Vol. 31, No. 3, 2001. pp. 295-303,

[12] Swamy, N., O. Kuljaca, O., and Lewis, F., "Internet-Based Educational Control Systems Lab Using Net-meeting”, IEEE Transaction on Education, Vol. 45, No. 2, pp. 145-151, May 2002,

[13] A. R. Al-Ali and M. AL-Rousan, 2004, "Java-Based Home Automation System", IEEE Transactions on Consumer Electronics, Vol. 50, No. 2,

[14] Stanciu, I., R., Ginga, Gh., Muntean, S., Anton, L., E., "Low-Speed-Small-Load Direct Torque Control Ripples Filtering", in Proceedings of the Romanian Academy Journal, Vol. 13, No2, 2011. pp. 125-132,

[15] Stanciu, R., Sorandaru, C., "A self-Configurable Real-Time Video Surveillance System with Distributed IP-Cameras", IEEE International Symposium on Logistics and Industrial Informatics, pp. 69-74, September, Smolenice, Slovakia, September, 2012. DOI: 10.1109/LINDI.2012.6319464,

[16] Ginga, G., Stuparu, A.,Bosioc, A., Anton, L.E., Muntean, S., "3D Numerical simulation of the flow into the suction elbow and impeller of a strorage pump", in Proc. of the 4th International Meeting on "Cavitation and Dynamic Problems in Hydraulic Machinery and Systems", Belgrade, Serbia, 2011. pp. 151-160

[17] Van den Braembussche, R.A., 2006, "Flow and loss mechanisms of centrifugal pumps", in Design and analysis of high speed pumps, Educational Notes RTO-EN-AVT, Neuilly-surSeime, France, Paper 12, pp. 1-26,

[18] Ginga, G., Stanciu, I., R., Muntean, S., Baya, A., Anton, L., E., “3D Numerical Flow Analysis and Experimental Validation into a Model Impeller of a Storage Pump", Proc. of the 15th Conference on Fluid Flow Technologies, vol. II, Budapest, Hungary, 2012, pp. 804-811,

[19] Ginga, G., "Experimental and Numerical Analysis of the Operating Centrifugal Pump Storage", PhD Thesis No. 123, "Politehnica" University of Timisoara, 2012. (in Romanian) 